\title{
Effective supervision in social work and social care
}

Professor John Carpenter and Caroline Webb, Bristol University Dr Lisa Bostock and Caroline Coomber, SCIE

\section{Key messages}

- Research has demonstrated that good supervision is associated with job satisfaction, commitment to the organisation and retention.

- Supervision appears to help reduce staff turnover and is significantly linked to employees' perceptions of the support they receive from the organisation.

- Good supervision is correlated with perceived worker effectiveness. There is some evidence that group supervision can increase critical thinking.

- Supervision works best when it pays attention to task assistance, social and emotional support and that workers have a positive relationship with supervisors.

- The emotionally charged nature of the work can place particular demands on people in the field. It is important to provide opportunities for reflective supervision.

- In an inter-professional context, workers relate job satisfaction and professional development to their supervisor's expert knowledge, regardless of whether respondents shared the same professional background.

- The impact of supervision on outcomes for service users and carers has rarely been investigated. Anecdotal evidence suggests that supervision may promote empowerment, fewer complaints and more positive feedback.

- Overall, the empirical basis for supervision in social work and social care in the UK is weak. Most of the evidence is correlational and derives from child welfare services in the US. 


\section{Introduction}

This research briefing provides an overview of the evidence concerning the value of supervision in supporting the practice of social care and social work. It is relevant to both children's and adult social care services and includes a consideration of supervision in integrated, multi-professional teams. While the focus is on social work and social care, some of the research reviewed includes participants from other professions such as nursing and psychology.

The briefing covers evidence on the use of different models of supervision and outcomes for workers, employers, service users and carers. It considers evidence on the costs of supervision and concludes with implications for policy-makers, practitioners, organisations, service users, carers and researchers.

\section{What is the issue?}

Learning from supervised practice is an essential component of the education and training of social workers. Through regular, structured meetings with a supervisor, students learn how to manage a caseload, apply theory and research evidence to practice, perform the key tasks of assessment, planning and intervention, and reflect on their own professional development. Supervision is also an opportunity to seek and receive emotional support for undertaking what can often be a demanding and stressful role.

Supervision is, according to Lord Laming, ${ }^{1}$ the 'cornerstone' of good social work practice - an opinion reiterated by the Monro Review. ${ }^{2}$ This perspective is an important alternative to the managerialist approach which, according to Noble and Irwin, ${ }^{3}$ is preoccupied with supervision geared toward efficiency, accountability and worker performance.

A survey undertaken for the Social Work Task Force indicated that social workers in England were receiving very variable access to supervision and that it was process-driven and overly focused on the management of cases at the expense of reflection and professional development. ${ }^{4}$ A related concern, expressed by Davys and Beddoe, ${ }^{5}$ is that rather than benefiting staff and ultimately service users, supervision:

becomes part of a system of surveillance of vulnerable and dangerous populations. ${ }^{5,}$, pp 220

The benefits of developing a positive supervision culture across wider social care and children's services are now widely recognised. National guidance is limited, but regulations such as the National Minimum Standards, linked to the Care Standards Act 2000 , require that supervision in care homes, for example, takes place six times a year and that it focuses on all aspects of practice, philosophy of care and career development. ${ }^{6}$ More recently in England this has been expressed as:

receiving appropriate training, professional development, supervision and appraisal. ${ }^{7}$

This research brief applies to both social care and social work whilst recognising that there is a gap in the research evidence relating to social care. Few studies overtly address the supervision needs and experiences of social care workers, although child welfare workers in the US may have no social work training, depending on State requirements.

Internationally there has been concern about the retention of social workers. This has long been a major problem in child welfare services in the US, with a number of initiatives designed to promote supervision, increase job satisfaction, decrease stress and burnout, and encourage workers to stay. Evidence from these initiatives features in this briefing.

In the UK, there has also been concern about the supervision of social workers and social care workers working in integrated and multi-disciplinary teams, ${ }^{8}$ where professionals 
may not be supervised by others from the same discipline. Does this matter, if the key ingredients are the same? Or are there some things, such as values and working practices, which are special to the individual disciplines and therefore to the professional development of their practitioners?

What then do we mean by 'supervision'? The primary functions are:

- administrative case management

- reflecting on and learning from practice

- personal support

- mediation, in which the supervisor acts as a bridge between the individual staff member and the organisation

- professional development.

Although terminology differs, these functions have consistently been identified in the practice literature. ${ }^{5,9,10}$ Authors often seek to highlight particular functions - for example, referring to 'case management supervision' and 'reflective supervision'. ${ }^{10}$ Authors with a behavioural health background ${ }^{11,12}$ and social workers in North America who provide clinical social work (i.e. with an emphasis on counselling and psychotherapy) refer to 'clinical supervision'. ${ }^{11,13-15}$ Definitions of reflective and clinical supervision are very close, essentially emphasising learning from case work with a view to professional development. Nevertheless, as the review by Spence et al. ${ }^{16}$ observed, there is a proliferation of models, but little evidence that these have had any significant impact on the practice of supervision or outcomes for service users. ${ }^{11}$

The method employed in supervision is primarily the one-to-one meeting with a supervisor - in the UK usually the worker's line manager. But this is not inevitably so: reflective and professional development supervision may be given by a senior practitioner or external consultant, and group supervision may also be used. We need to understand which of these methods is most useful, for whom and in what circumstances.

\section{Why is it important?}

The overall aim of professional supervision should be to provide the best possible support to service users in accordance with the organisation's responsibilities and accountable professional standards. Organisations are likely to achieve this aim through workers who are skilful, knowledgeable, clear about their roles and assisted in their practice by sound advice and emotional support from a supervisor with whom they have a good professional relationship. The secondary aim should be for the wellbeing and job satisfaction of workers, not simply because satisfied workers may be more likely to remain in their jobs, but because a duty of care for staff working in difficult and challenging roles is important in its own right.

Despite the many of models of supervision, few, if any, are based on empirical research. This is ironic since many supervisors actively seek to promote evidence-based practice. It is widely assumed by policy-makers, educators and practitioners that supervision is a good thing. But does the available research support this assumption? This research briefing aims to assess the evidence. It begins by summarising what we know.

\section{What do we know already?}

The search identified three reviews of the literature concerning the outcomes of supervision for social workers and equivalent professionals. The narrative review by Spence et al. ${ }^{16}$ examined research on clinical psychology, occupational therapy and speech pathology as well as social work. It was not clear whether supervision had any effect on workers' practice or whether it led to improved outcomes for service users. There was some evidence to suggest that directive, as opposed to unstructured, approaches were preferred by less experienced practitioners and also by the more experienced when faced with new challenges. All supervisees preferred 
a supportive style of supervision. The authors observe that supervisors reported little or no training in how to supervise. Nevertheless, those from the different disciplines engaged in very similar supervision practices. Although supervisors claimed to adapt their supervision styles to the needs of individual supervisees, the majority did not appear to do so.

Bogo and McKnight ${ }^{17}$ reviewed 13 peer-reviewed articles from the US based on 11 separate studies. Like Spence et al., ${ }^{16}$ they found little evidence on the outcomes of supervision, but conclude that there is emerging evidence about the aspects of supervision valued by supervisees - specifically availability, positive relationships, mutual communication, support and delegating responsibility. Skilful supervisors with expertise who were able to provide practical support were particularly appreciated.

Most recently, Mor Barak et al. ${ }^{18}$ conducted a meta-analysis of data from 27 papers which provided information about the relationships between three dimensions of supervision and various outcomes for social workers, child welfare and mental health workers. The dimensions were:

- task assistance, defined as the supervisor's ability to provide tangible, work-related guidance

- social and emotional support in responding to emotional needs, including stress

- interpersonal interaction, which reflects the supervisee's perceptions of the quality of the relationship and the extent to which this has helped them be more effective in their work.

These dimensions were all positively and significantly associated with beneficial outcomes for workers, including job satisfaction, commitment to the organisation, wellbeing and perceived effectiveness. Conversely, they were negatively associated statistically with detrimental outcomes such as stress, burnout and intention to leave.
The evidence for Mor Barak's meta-analysis was drawn from correlational and cross-sectional studies in which a large number of variables are investigated for their statistical associations with outcomes for workers. In other words, this evidence is not causal. It does not, and cannot, prove that the observed effects at the time the data were collected can be attributed to the outcomes of supervision. Nevertheless, this research provides good circumstantial evidence for the effects of supervision on workers, and also helpful definitions of key dimensions of supervision and a framework for analysing outcomes.

\section{What this research briefing adds}

The review undertaken for this research briefing updates the evidence base to 2012 and extends previous reviews by focusing on outcomes for organisations and service users in addition to workers. The briefing extracts information about the nature and focus of the supervision as described in the studies. It also assesses the quality of the evidence and its relevance for social work and social care practice in the UK.

The briefing identifies empirical studies that report on the association between the process of supervision and outcomes for service users, workers and organisations. Intervention studies are included. However, due to resource constraints, studies addressing the perspectives of supervisors have been excluded. The methods used to identify and organise material were developed by the Social Care Institute for Excellence (SCIE). These involved undertaking systematic and reproducible searches of the research literature, identifying relevant studies and assessing their quality. Empirical data was extracted using a structured pro-forma which focused on various outcomes of supervision.

\section{The literature}

The briefing aims to provide a signpost for further reading, rather than a definitive account of 'what 
works'. It is based on papers published in peer reviewed journals only between 2000 and 2012 . Fifty papers were identified, reporting 48 separate studies. The literature is predominately US-based, ${ }^{19}$ with another seven studies reporting data from Australia, Canada and Israel. Just three studies were based in the UK.

The majority of papers focus on social work with children and families, ${ }^{20}$ with just four considering adult social care. Most studies ${ }^{21}$ are cross-sectional surveys and report only correlational evidence in which supervision figured as one factor among many that were associated with practitioners' job satisfaction, stress, retention and intention to leave. Only four studies report the results of interventions using supervision to improve outcomes.

Just two papers report on associations between the process of supervision and outcomes for service users. No study reports on the perspectives of service users, hence the literature is silent on users' and carers' views on supervision.

Two papers specifically focus on inter-professional working within integrated teams, of which both concern practice with adults. One, focused on children and families, briefly addresses intra-agency working.

Both social work and social care staff are included in this review. However, it is not always made explicit whether some papers refer to staff qualified in social work, unqualified workers or those with other related qualifications. Because a substantial amount of research literature is from the US and from child welfare services, it is important to note that the educational requirements for child welfare workers in that country vary by state and even region and municipality, meaning that many child welfare workers have no social work qualification or training. Hence, supervisors in these settings often have to teach basic social work knowledge, values and skills which it is likely social workers in the UK would already possess. There is just one study which explicitly examines the perspectives of social care staff, in this case home care workers (HCWs) in Northern Ireland.

A significant problem faced by the reviewers was that few papers provided information on the nature, quality and regularity of supervision. This, together with the fact that most of the evidence is correlational and drawn extensively from services in the US, makes it impossible to draw definitive conclusions about the outcomes of supervision in the UK. Nevertheless, this briefing does identify a range of possible outcomes for practitioners, employers and service users and assesses the extent of evidence for each.

\section{What does the research show?}

\section{Models of supervision}

Few of the 50 studies reviewed provide information about the nature of supervision. This was noticeably the case in the majority of studies included which investigated the statistical associations between supervision and outcomes for workers. This lack of specificity unfortunately limits their usefulness.

In general, the majority of studies concerned one-to-one supervision. Although it was not clear whether this was provided by the worker's line manager or another person, the assumption seems to be that it was the former. Of the few studies which evaluated an intervention to implement a new or enhanced approach to supervision within an organisation, three describe the model of supervision in some detail. $13,22,23$

These interventions all took place in child welfare services in the US. The first three involved groupbased supervision, either alone or in combination with individual supervision. Where the focus of supervision was described, it was generally 'reflective' or 'clinical' supervision as opposed to 'administrative' or 'case management' supervision. 
Smith et al. ${ }^{23}$ report on the Integrative Supervision Model (ISM) in Georgia, US. The four stages of ISM focus on case management, educational/professional development, clinical skills/reflective problem-solving and emotional support. Experienced supervisors were bought in and trained in ISM. The length and frequency of the group supervision sessions is not described, but the project lasted around 18 months. A pilot study employing pre-post measures with 17 social workers indicated a statistically significant change in participants' perceived levels of professional knowledge and skills. This finding was supported by focus group data from social workers and supervisors.

There were no studies comparing the outcomes or cost-effectiveness of different models of supervision. However, in a pilot study, Lee et al. ${ }^{24}$ assessed the job satisfaction ratings of mental health workers in one service agency in the US and conclude that participants who perceived their supervision to involve mentoring relationships were more satisfied with the work itself, their supervision and their co-workers than staff who did not.

Mentoring, defined as assigning tasks, teaching the job, giving support, and providing inspiration and advocacy, was also identified as a key component of job satisfaction for supervisors in a large-scale survey of child welfare services in the US. ${ }^{25}$

\section{Outcomes for workers}

The importance of supervision to outcomes for workers was the focus of most of the studies reviewed. The conceptual model by Mor Barak et al. ${ }^{18}$ identifies both beneficial and detrimental outcomes associated with the dimensions of supervision and provides a framework for this section.

\section{Job satisfaction}

The quality of supervision is consistently associated with positive worker outcomes, with a significant number of papers addressing the impact of supervision on job satisfaction. 12,14, 15,24,26-33 Job satisfaction coheres around the following three themes:

- structure, focus and frequency of supervision

- task assistance (supervisor's tangible, work-related advice and instruction to a supervisee)

- support to access resources for service users.

Structure, focus and frequency of supervision Where reported, greater frequency of supervision is associated with higher levels of satisfaction, with one study reporting a minimum of two hours per week as a perquisite to job satisfaction and retention for urban child welfare workers in the US. ${ }^{26}$

In a qualitative study that attempted to get to grips with the structure, focus and frequency of supervision, Bogo et al. ${ }^{14,28}$ explored the experiences and perceptions of frontline health and social care staff in relation to clinical supervision and best practice. The context was the merger of two addiction services and two mental health facilities to create the Centre for Addiction and Mental Health (CAMH) in Toronto. Programme management was introduced to improve patient care, meaning that professionals no longer necessarily received supervision from someone of their own professional background.

Bogo et al. found that job satisfaction and professional development, regardless of whether respondents shared the same professional background, were related to the following important components of supervision:

- that it was regular

- that it was provided by those with expert knowledge and clinical intervention skills for the specific client population

- that it was able to teach new, effective treatment methods and that there was reciprocity and active involvement from supervisees. 
Staff suggested that the emotional climate of supervision - meaning a 'safe, confidential space' - was key in helping them to process the personal impact of practice experiences. In contrast, when supervision was focused on administrative issues or performance management and productivity, it was experienced negatively as being critical and undermining of staff confidence.

\section{Task assistance}

Task assistance involves a supervisor's tangible, work-related advice and instruction to a supervisee and focuses on training, skills and solutions for practice. ${ }^{18}$ It is primarily related to job satisfaction in this review, underlining its importance to positive outcomes for workers, supporting them to perform effectively. Task assistance was of particular importance to workers in terms of role clarity, supporting them with perceived role competence and with task knowledge and problem-solving. ${ }^{27}$

In Australia, Kavanagh et al..$^{12}$ examined the frequency and characteristics of practice-related supervision in allied health staff, including social workers in mental health services across Queensland. The research reports supervision as largely individual and face-to-face, with some by teleconferencing in rural and regional areas. There was a monthly median of two hours. Almost all respondents had a supervisor from their own profession and 45 per cent had multiple supervisors. The primary focus of supervision was on practice improvement rather than therapeutic discussion for the supervisee. Both supervisors and supervisees reported the main focus of the supervision as:

- discipline-specific competencies (56 per cent supervisors; 46 per cent supervisees)

- generic practice skills (15 per cent supervisors; 25 per cent supervisees)

- personal issues and career development (8 per cent for both supervisors and supervisees).

Supervisees reported little observation of their clinical practice, practice skills or use of audio-visual aids. Problems identified with the delivery of supervision included:

- high clinical workload

- availability of supervision partner and sessions not frequent enough

- insufficiently experienced supervisors

- lack of guidelines

- a need for training in supervision skills.

Kavanagh's study goes beyond the description of supervisory practices and characteristics and examines which features were associated with perceived impact on practice. Analysing responses from supervisees only, due to supervisors rating multiple relationships, the study found:

- satisfaction with supervision and positive attitudes to supervisors were strongly associated with perceived impact on practice

- a positive relationship between frequency of contact with supervisors from the same discipline and perceived impact, but not with cross-professional supervision

- that giving priority to discipline-specific skills was strongly associated with impact, but time spent on generic skills was not.

The authors conclude that:

from a management point of view, it suggested that supervision may contribute to better patient care and to staff retention. A need for a more targeted approach to skills acquisition was identified, as was a discipline-specific focus in sessions. Positivity of the supervision relationship emerged as a key feature of effective supervision, both in terms of its impact on practice and on job satisfaction.

\section{Accessing resources}

In a study of home health social workers in the US, Egan and Kardushin ${ }^{30}$ report on task assistance in relation to accessing resources for service users and its impact on job 
satisfaction. The helpfulness of 'administrators' (i.e. budget-holders) in resolving difficulties between patient access to services and financial priorities contributed significantly to greater job satisfaction. The authors conclude that 'administrative' supervision, whereby supervisors help their staff access resources to meet patient need and thus resolve their own ethical conflicts or uneasiness about not being able to offer the services needed, is more important than 'emotional' supervision focused on professional development and mentoring in this cost-conscious context.

\section{Supervision of the worker}

Social work and social care are conducted through personal relationships and interactions, with or without practical support or personal care. They place particular demands on staff and it is the employer's responsibility to ensure this dimension of supervision is provided as part of their duty of care. Outcomes should include making sure that practice is safe, from the point of view of people using services and their families as well as workers, through personal risk assessment, debriefing after difficult situations and reviewing decisions for learning. Outcomes of supervision should also concern consideration of the worker as a professional practitioner, entitled to development and learning opportunities, status and standing alongside other professionals, and capable of representing the agency in joint and integrated working. Positive outcomes for workers reported in the literature include:

- social and emotional wellbeing

- improved self-efficacy and sense of empowerment

- organisational commitment and intention to stay.

Detrimental outcomes include:

- stress

- burnout

- role conflict

- intention to leave.
Social and emotional wellbeing

Supporting the social and emotional needs of workers entails listening to them attentively as they discuss job difficulties and making empathic comments. It may also include relating to the emotional needs of workers when they feel overwhelmed, stressed or confused about their work. ${ }^{18}$

In a study of job satisfaction among hospice inter-disciplinary team members in the US, Deloach ${ }^{29}$ reports qualitative responses from open-ended survey questions on the aspects of supervision that staff found most supportive. Social and emotional support figured highly. For social workers, being supportive came in the form of feeling valued as a unique member of a specific discipline, being supported in clinical decision-making and supportive comments by supervisors that 'back you up'.

\section{Self-efficacy and empowerment}

A perceived sense of control, or self-efficacy, is also associated with job satisfaction for workers. ${ }^{34}$ Where supervisors are socially and emotionally supportive to supervisees, studies show that self-efficacy is related to job satisfaction ${ }^{15}$ and intention to stay. ${ }^{24}$ In Cearley's study $^{35}$ of child welfare workers in the US, supervisors' empowering behaviours significantly affected workers' sense of empowerment, specifically increasing their ability to make decisions. These quantitative associations were confirmed with qualitative data - for example, one worker stated that her supervisor:

allows workers to make their own decisions and she supports those decisions after they are made.

\section{Organisational commitment and intention to stay}

Organisational commitment to the development of supervisory practice, ${ }^{13}$ the willingness of supervisors to help employees carry out their jobs effectively and provide aid in stressful situations, ${ }^{25}$ and whether supervisees feel emotionally supported by supervisors ${ }^{31,36-38}$ are 
all associated statistically with workers' decision to stay employed.

The degree to which employees feel supported by their supervisor affects their emotional satisfaction with the job and contributes to the appraisal of how the organisation values them and cares about them. In Landsman's ${ }^{31}$ study of organisational commitment by child welfare workers, it was found that supportive supervision was associated with both job satisfaction and perceived organisational support.

Kavanagh et al. ${ }^{12}$ found that while there was no association between the amount of supervision and job satisfaction or intention to stay, there was a link with positive attitudes to supervisors and high supervision impact. Relationships that were characterised by accessibility, empathy and praise appeared especially important for retention. Retention and intention to stay are also related to access to supportive peer relations at work. ${ }^{39-41}$

\section{Stress, burnout and role conflict}

Supervisory support has clear associations with worker stress, burnout and role conflict. Lack of emotional support, inadequate supervision and feeling out of one's comfort zone are associated with higher burnout. ${ }^{42}$ Job-relevant communication, another facet of task assistance, is also reported as important to positive worker outcomes.

Kim and Lee $^{32}$ used statistical modelling to investigate the effects of different types of supervisory communication on burnout and intention to leave among 211 social workers in health or mental health settings in the US. 'Supportive relationship communication', defined as informal and supportive interaction between supervisors and social workers, appeared to reduce worker stress and indirectly reduced burnout and intention to leave. 'Job-relevant communication', defined as performance feedback, information about rules, policies, work schedules and assignments, task-specific instructions and goals, was found to be more effective if a supervisor and a worker interact with each other in a supportive way, directly reducing intention to leave. Professional development through supervised practice was also associated with workers' attachment to the organisation. Where frontline workers were readily able to communicate their opinions and feelings to management, this reduced the role of stress on burnout.

Burnout is also associated with workers' perception of their relationship with the supervisor, not just whether they received help or support. ${ }^{18}$ In Mena and Bailey's ${ }^{33}$ study of child welfare workers in the US, workers' sense of rapport within the supervisory relationship was related to job satisfaction. Conversely, where workers reported feeling negative about rapport, this was associated with both emotional exhaustion and depersonalisation, whereby workers feel detached, no longer see themselves as valuable and lose track of their personal needs.

In another US-based study of child welfare workers, Boyas and Wind ${ }^{43}$ found that emotional exhaustion was significantly higher for workers receiving increased supervisory support. This counterintuitive finding highlights the difficulty of drawing conclusions from correlational evidence. Having controlled for key individual characteristics, such as age, tenure and job title, Boyas and Wind speculate that emotional exhaustion is higher among experienced workers who have greater involvement in difficult decision-making processes and handle the more complex child protection cases.

Supervising the emotionally charged nature of child protection work requires a refocusing of supervision so that it explores the impact of the thoughts and feelings of workers on practice. In a small-scale qualitative study by Gibbs ${ }^{44}$ of child protection workers in two rural regions in Victoria, Australia, it was found that the model of supervision gave insufficient attention to the emotional intrusiveness of the work.

Respondents reported that the overriding priority for supervisors was to ensure the work was 
completed and conformed to acceptable standards, at the expense of reflection on practice, discussion about high workloads, stress and anxiety. This meant that workers experienced role ambiguity, stress and concern about things going wrong. Gibbs concludes that supervision must:

- convey the value of workers

- explore feelings and thoughts in action and perception

- take account of adult learning theory and the role of the supervisor in promoting effective learning by staff.

\section{Intention to leave}

Nine papers look at the association between supervision and intention to leave. Findings are contradictory. Two papers by Strolin-Goltzman ${ }^{45,46}$ report that supervisory support does not predict intention to leave, with a related paper by $M c G o w a n^{47}$ suggesting that career satisfaction was the most important factor determining intention to leave. In contrast, six studies found that supervision plays an important role in determining whether social workers consider leaving their jobs. ${ }^{32,39-41,48-50}$ Fakunmoju ${ }^{49}$ found that gender played a moderate role in regard to supervisory support and intention to leave, with men more likely to report higher intention to leave than women in relation to low supervisory support. However, it is difficult to unravel which particular aspects of supervision make a difference to workers' intention to leave because data on the structure, focus and frequency of supervision is rarely reported.

In one of the few UK-based studies, and the only study that explicitly addressed social care workers without a social work qualification, Fleming and Taylor ${ }^{51}$ looked at the retention of home care workers (HCWs) in Northern Ireland. Based within an integrated health and social care service, this small-scale study surveyed $45 \mathrm{HCWs}$ and focus groups to explore the growing problem of retention of HCWs from their own perspective. On the whole, $\mathrm{HCW}$ s were positive about supervision, but a significant minority identified the need for more support, better communication and more responsive out-of-hours and emergency systems. Emergencies were a particular crunch point, with about a third of staff saying that they sometimes or never felt supported.

The type of support thought to be beneficial included:

- extra time and support available in a crisis (e.g. death of a client or preparing for hospital)

- information about clients requiring temporary cover

- better out-of-hours contact arrangements with supervisors

- weekly contact from a supervisor

- more consideration given to HCWs regarding client care

- a stand-by HCW available in the evenings and weekends to assist with emergencies.

\section{Outcomes for organisations}

Many of the studies also consider outcomes of supervision that are beneficial to organisations. Supervision focused on task assistance for the worker may improve performance, while supervision which provides social and emotional support may reduce staff turnover.

\section{Job performance}

Increasingly, supervision of social workers has become focused on performance management, ensuring that organisational procedures have been followed and that workers are practising within agency expectations. However, there is very little evidence that supervision affects performance.

Kavanagh et al. ${ }^{12}$ found that satisfaction with supervision and positive attitudes to supervisors were strongly associated with perceived impact on practice, but only if supervisors were from the same profession. This, they suggest, may be 
because certain features of supervision are important, such as direct instruction and skills acquisition, thereby emphasising the task assistance function.

The study by Smith et al. ${ }^{23}$ evaluated the impact of the Integrative Supervision Model (ISM) described above. There were changes in participants' self-perceived levels of knowledge and skills during the project, and it was believed that this had a beneficial impact on service user outcomes, but there was no direct evidence to support this assertion.

While the evidence suggests an association between supervision and perceptions of job performance in general, this review found no studies evaluating the impact of supervision on specific aspects of job performance. Neither was there sufficient detail on the supervision processes to draw any conclusions about how supervision positively affects job performance. It may be that the task assistance function of supervisors has a direct impact, but equally, increased worker perceptions of job performance may also be an indirect effect of increased self-efficacy as a result of supervision. Once again, this is a limitation of correlational evidence.

\section{Workload management}

Just two studies consider the potential buffering effect of supervision on workload management, although this is not addressed in any detail and the studies do not provide detailed descriptions of caseloads. In a US study of child welfare workers, Juby and Scannapieco ${ }^{52}$ found that staff who received more support from their supervisors saw their work as more manageable. They suggest that this may reflect the task assistance function of supervision as it increases workers' skills and knowledge by providing education and training. Collins-Camargo and Millar ${ }^{54}$ report on the outcomes of clinical supervision projects with a particular emphasis on the development of learning cultures which promote self-reflection, evidence-informed practice and outcomes-focused approaches to working with families. They found that workers' time management improved as a result of the new supervisory processes, however it is not clear from this research why supervisory practice resulted in these impacts.

\section{Case analysis and planning}

Four studies consider the impact of supervision on case analysis and planning. In one of the few intervention studies found in this review, Leitz ${ }^{22}$ evaluated a group supervision project designed to develop critical thinking skills (termed a 'supervision circle') for over 300 child welfare workers in Arizona, US. This one-year project began with five training sessions for supervisors who then provided group sessions for five to seven workers on a fortnightly or monthly basis. The focus was on peer case review and other critical thinking exercises.

There was a statistically significant increase in perceived levels of critical thinking among the participants. The quality of the relationship between participants and lead supervisor, along with the extent of participation in group supervision, predicted the level of critical thinking at the conclusion of the project. The number of hours spent in supervision did not significantly predict perceived levels of critical thinking, which suggests that it may be quality of supervision rather than quantity which is important.

In a further study which considered therapists working with survivors of domestic abuse in Israel, Ben-Porat and Itzhaky ${ }^{27}$ found that satisfaction with supervision correlated positively and significantly with two components of role competence: general competence and knowledge and problem-solving.

Improved case analysis and planning was also a theme in the study by Smith et al. ${ }^{23}$ Using the ISM, these researchers found that workers reported they were able to apply ideas from their supervision group directly to their caseloads, as well as being able to identify specific clinical skills used by other group members. Collins-Camargo and Millar ${ }^{54}$ also report improved case analysis 
via critical thinking and realistic targeting of interventions, although no further in-depth analysis of these results is offered.

\section{Actual turnover and retention}

In this review, workers' intention to leave is considered as an outcome for the individual worker and was therefore discussed in a previous section. Actual turnover - and its converse, retention - are considered as (detrimental) outcomes to organisations.

Fourteen studies examined the links between supervisory support and actual turnover and retention rates. The general consensus is that good supervision can help workers to stay in their jobs, while leavers often cite poor supervision as a reason for having left.

In a study of US child welfare workers who had recently left their jobs, Gonzalez et al..$^{55}$ report that 29 per cent cited problems with supervision as their reason for leaving ( 65 per cent of these due to 'poor supervision' and 35 per cent due to their relationship with their supervisor), while 25 per cent said changes in supervision would have made them stay (including more supervisor availability).

\section{Quality of supervision}

Differences between stayers and leavers in the quality of supervision received were apparent in research by Dickenson and Perry. ${ }^{56}$ They report that those workers remaining in public child welfare rated their supervisors at a significantly higher level in terms of willingness to listen to work-related problems, the extent to which they could be relied upon 'when things get tough at work', and helping workers get their job done. Significant differences were also observed in terms of stayers' views on the skills and characteristics of their supervisors. This staff group rated their supervisors as more competent, more concerned with staff welfare, more likely to show approval of a good job done, more likely to help in completing difficult tasks and more likely to be 'warm and friendly' when workers experience 'problems'.
Similarly, Maertz et al. ${ }^{57}$ report that stayers gave higher ratings than leavers on how their supervisor facilitated their learning and enthusiasm for the job as well as significant differences in the average number of hours spent with their supervisor each month.

\section{The supervisory relationship}

One notable aspect of supervision associated with turnover and retention rates concerns the supervisory relationship. Yankeelov et al. ${ }^{58}$ and Gibbs $^{44}$ both report that those workers who stayed were more attached to their supervisors than those who had left; some staff were able to vividly describe the experience of being supervised by one particular person, even if this was some years previously. This suggests that stayers feel a sense of security in their relationships with their supervisors and that this relationship is highly significant to them.

In a qualitative comparison of child welfare workers in the US conducted by Morazes et al., 59304 stayers and 82 leavers found no differences in terms of dedication to children and families and social work values. While all participants acknowledged workload and stress as job challenges, stayers illustrated experiences that buffered job pressures, particularly encounters with supportive supervisors. As one stayer reported:

I think I stay [because] ... my unit is good, my supervisor is excellent and that really makes a difference. I think if I had a supervisor I wasn't getting along with or wasn't helping me I would really want to leave. You need that support while you are here because it's a hard job and you are dealing with difficult things ...

This study goes on to suggest that leavers tended not to experience these buffers, although they recognised the value of them, and they were more likely to describe experiences in which they actually felt worn out by the same influences which stayers had experienced as supportive, such as supervisors. 
Other studies report mixed findings regarding the link between supervision and retention (as do the studies reporting on workers' intention to leave). Faller et al. ${ }^{60}$ found that workers who indicated their supervisor made life difficult were significantly more likely to have left their job. Yet not all aspects of supervisor support influence staff retention, and it was found that the supervisory roles of providing useful information when needed, or helping with new or unfamiliar tasks, were not significantly associated with retention. Strolin-Goltzman ${ }^{45}$ found that there were no significant differences in supervisory factors between organisations classed as having high versus low turnover rates, thereby contradicting prior research findings. The association between supervision and retention is likely to depend on both the type of supervision provided and whether it is provided in the context of a supportive relationship.

\section{The long-term impact of supervision}

There are also questions regarding the long-term impact of supervision on staff retention rates. Renner et al. ${ }^{13}$ found that at one-year follow-up after implementing a state-wide supervision plan among child welfare workers in Missouri, US, retention rates improved, although only a little (from 75 to 78 per cent), but by two years these had fallen back to 74 per cent.

It should also be remembered that high turnover among supervisors could leave frontline staff quite vulnerable if the job commitment of staff is tied closely to perceptions of supervisor support - as the literature suggests. ${ }^{38}$ In the UK, Holmes et al. ${ }^{61}$ note that two local authorities identified particular difficulties recruiting team managers, and that these posts were seen as critical in supporting teams as well as driving improvements in practice.

\section{Perceived organisational support (POS)}

Perceived organisational support (POS) is the idea that employees form a global belief concerning the extent to which the organisation values their contributions and cares about their wellbeing. Research into POS is increasing, particularly in relation to its links with staff turnover. In this review, seven studies consider the relationship between supervision and POS.

In the US, Maertz et al. ${ }^{57}$ suggest that POS may remain fairly stable over time, and may be less salient to employees than supervisory support, because supervisors have more regular contact with workers. However, for employees who are not receiving effective supervisory support, POS becomes more important as they turn to the organisation instead. This research concludes that employees require a certain level of support and this may be supplied by the supervisor, the organisation itself or a combination.

Landsman $^{31}$ found that supervisory support significantly affected ratings of POS, and concludes that the degree to which employees feel supported by their direct supervisor affects their emotional satisfaction with the job and contributes to their appraisal of how the organisation values them and cares about them. Gibbs $^{44}$ also reports that for some staff a lack of effective supervision to enable them to manage the emotional demands of the job left them believing they had been poorly supported within the organisation, but for others this response was internalised and workers saw themselves as having failed in what they were doing when things had gone wrong.

Collins-Camargo and Millar ${ }^{54}$ report that overall changes in supervisory practice had a positive impact on worker perception of organisational culture, and Collins-Camargo and Royse ${ }^{53}$ found that effective supervision was highly correlated with staff perceptions of an organisational culture that promoted evidence-based practice.

Bourn and Hafford-Letchfeld ${ }^{62}$ suggest that frontline managers in the UK have a key role in mediating organisational culture. Their small-scale qualitative study of recordings and transcripts of trainee managers' supervisory sessions indicates that supervisors frequently use humour, somewhat ironic apologies or other tactics for diffusing conflict and 
aggravation or for gaining compliance with the implementation of otherwise unwelcome procedural changes. The authors conclude that the supervisory relationship has an important mediation function: it is a channel through which organisational culture is conveyed to supervisees as well as providing an opportunity for staff to convey information upwards to the organisation.

Chenot et al. ${ }^{37}$ suggest that organisational culture only impacts on workers in the early phase of their career, after which staff become accustomed to agency norms, which could be detrimental in a negative organisational culture. Maertz et al. ${ }^{57}$ caution that distinctive attachments to supervisors may even increase voluntary turnover when a loyalty-inspiring supervisor leaves.

\section{Supervision in multi-disciplinary settings}

The qualitative study of supervision in an inter-agency setting carried out by Bogo et al. ${ }^{14,28}$ is discussed above. They focused on workers' views about supervision arrangements following the amalgamation of two mental health and two addiction services in Canada. Programme management meant that professionals no longer necessarily received supervision from someone of their own professional background.

\section{Mixed reactions were found. Some staff} reported that supervisors would not discuss clinical issues or would focus solely on performance management, whereas other staff felt more positive. Overall, workers valued supervisors who attempted to understand the frameworks of their professions, although they missed the connection with their professional discipline and the ability to 'talk in their own language'. Some social workers also:

experienced tension between the ... focus on client/public safety, and social work values of being non-judgemental and promoting empowerment.
Staff found the new inter-professional teams a valuable source of support which provided spontaneous and informal feedback in the face of critical and emotionally provocative experiences. These views did not appear to be related to their perceptions of the supervisor, suggesting they are separate constructs.

In the second paper, Bogo et al. ${ }^{28}$ report that participants perceived safety and trust to be more important than whether the supervisor was from the same profession (although as noted in Kavanagh et al.'s' ${ }^{12}$ paper, having a supervisor from the same profession was important for job performance). Almost all participants agreed that the key elements of valued supervisors are their clinical expertise and their ability to provide new and relevant practice knowledge and promote learning in a respectful and safe way. These were more important than their professional affiliation. However, some participants did comment that where they were the lone member of the profession on the team, meetings with others from their own profession were important as inter-professional supervision may not include profession-specific work.

\section{Inter-agency working}

No studies were found in relation to the impact of supervision on inter-agency working. Although Collins-Camargo and Millar ${ }^{54}$ report that the new supervisory processes used in the clinical supervision projects resulted in improved intra-agency working (same agency but between counties), as well as increased agency credibility from external sources, no analysis of why this occurred is offered.

\section{Outcomes for service users and carers}

The impact of supervision on outcomes for service users and carers has rarely been investigated. In part, this may reflect the difficulties of unravelling the distinct impact of supervision on service user outcomes, but may also reflect a preoccupation with outcomes for workers and organisations. Where evidence exists, the outcomes are defined by professionals, 
making it difficult to assess the impact of supervision on outcomes that matter to service users themselves, which may differ from policy and practice imperatives. This means that the literature presents only a partial picture of the outcomes that count, with no attention paid to the potential differences between professionals and users. It also means that any changes to the supervisory process are not informed by the perspectives of service users and carers and miss a crucial aspect of understanding how supervision impacts on practice.

\section{Empowerment, participation and positive feedback}

Where studies have attempted to assess the impact of supervisory focus on worker satisfaction and ultimately outcomes for children and families, positive outcomes for users have been identified. These include promotion of empowerment and user participation, fewer complaints and an increase in positive feedback.

Collins-Camargo and Miller ${ }^{54}$ looked at the introduction of new supervisory processes in four state public child welfare agencies in the US. This involved a shift from supervision focused on administrative and crisis-driven approaches to clinical supervision designed to promote:

- a supportive organisational culture

- an outcomes-oriented approach

- enhanced workers' ability to think critically and make good decisions regarding assessment of their cases

- evidence-based practice.

Supervisors participating in this project were asked to assess the impact on service users of changes to supervisory practice through discussion in focus groups. This data was therefore anecdotal. The following areas were identified:

- active participation in services

- increased engagement in case planning
- families demonstrating positive empowerment and a desire for positive change

- fewer complaints and more positive feedback from users.

The authors do not offer an in-depth analysis of why changes to supervisory practice might have had the claimed impacts.

There was also a perception among supervisors that cases were moving more quickly, and an anecdotal belief that children were going home sooner or not being removed from their homes in the first place. Quantitative data was not analysed to support this. Quotes suggest that supervisors attribute this to closer working relationships with families, addressing issues as they came up rather than leaving cases to drift. However, the anecdotal nature of this evidence means that claims made about the impact of this intervention on service users should be treated very cautiously.

\section{Placement safety and family functioning}

In a paper exploring how organisational characteristics have the potential to have a positive or negative impact on outcomes for service users, Yoo $^{63}$ looked at the role of the case manager as the service provider with most contact with users, and who acts as a bridge between clients and agency.

Exploring a family preservation service in the US, Yoo found that while the agency was successful in terms of placement safety and family functioning, the organisational climate was perceived by workers as not conducive to effective practice.

Drawing on secondary data from the agency's programme evaluations, Yoo found that at completion of the programme, out of 108 client families, 101 had children remain at home with no safety issues present and family functioning increased significantly. However, unravelling the role of supervision as a contributor to this success is complicated. Case managers 
reported various organisational challenges, such as low pay and benefits, harsh working conditions and poor leadership as having a negative effect. They also reported organisational characteristics perceived as positive strengths, such as commitment to client families and the support they receive from supervisors and co-workers.

While case managers were unable to identify in specific terms what and how organisational characteristics impacted on their work with service users, the author notes that:

the case managers' performance in contributing to positive outcomes for their clients seems to occur despite their dissatisfaction with many aspects of their organisation, including compensation, work conditions and leadership.

\section{Cost and cost-effectiveness}

There was only one study identified which considered the costs of supervision. In this UK-based paper examining children's social care services, Holmes et al. (cited in Munro²) calculated that the unit cost of a supervision session lasting 1.5 hours is $£ 87$ per frontline social worker. They note that if the frequency of supervision sessions is increased from monthly to fortnightly (to allow greater time for other aspects of supervision as well as case management, for instance), this would result in an annual increase in costs of $£ 1,217$ (per worker), and for a referral and intake team with five social workers and three family support workers an additional cost of $£ 9,408$. While any paper offering an estimation of costs is noteworthy, without a clearer idea about the benefits or outcomes of supervision this data remains somewhat arbitrary. However, since some studies suggest that supervision is associated with staff retention, and as turnover results in increased organisational costs, reduced effectiveness and poorer outcomes for service users, further consideration of the cost-effectiveness of supervision is crucial.

\section{Implications from the research}

This review of the research has identified a substantial volume of evidence that the provision of supervision is associated with positive outcomes for workers (e.g. job satisfaction) and organisations (e.g. job retention). However, there is as yet little evidence that the implementation of structured supervision can improve these outcomes, and the evidence for its effects on workers' practice is weak. Further, it has not yet been demonstrated that supervision directly affects service user outcomes (e.g. child safety, mental health, reablement).

\section{Implications for the policy community}

The present emphasis in policy-making on the implementation of reflective supervision for newly-qualified social workers is supported by the evidence in this review. Supervision is also associated with beneficial outcomes for more experienced workers and policy-makers should continue to promote its use more widely.

Supervision may enhance worker effectiveness, maintain job satisfaction and promote retention of staff.

\section{Implications for practitioners}

Supervision works best when it pays attention to task assistance, social and emotional support and a positive interpersonal relationship between supervisors and supervisees. In particular, task assistance and the importance of supervision in the acquisition of new skills and problem-solving are valued by workers. This is true for both relatively inexperienced practitioners and more experienced workers when learning new skills, working with new service user groups, in new multi-disciplinary teams or dealing with critical or complex situations.

Given the evidence that supervision is associated with job satisfaction and protects against stress, practitioners should insist that good supervision be provided by their employers. The emotionally charged nature of the work places particular 
kinds of demands on people working in the field which need to be contained by the organisation. This means moving beyond a focus on task and prescription, and providing opportunities for reflective supervision.

\section{Implications for organisations}

Supervision plays a key role in social work and social care. Effective supervision is a crucial element of an organisation's duty of care to its employees, and to the users and carers it serves. Supervisors occupy a unique role whereby they communicate the organisation's duties and priorities to the worker, and also feed back workers' comments to the wider organisation.

The studies in this research briefing have shown that the task assistance, emotional and support functions of supervision have positive effects on a variety of organisational outcomes. Effective supervision results in more positive perceptions of job performance and a greater ability to manage workloads, while employees' case analysis and planning skills are honed. Supervision also appears to help reduce staff turnover and is significantly linked to employees' perceptions of the support they receive from the organisation.

\section{Implications for service users and carers}

It is reasonable to assume that well-supervised social care workers who are supported both in their roles and tasks and also in dealing with the emotional aspects of their jobs will be able to provide a better service and improved outcomes for users and carers. However, at present, the evidence to support this view is anecdotal and therefore weak.

Service user-defined outcomes may differ from policy and practice imperatives. They are a crucial aspect of understanding the effectiveness of services from the perspectives of those who use them and consequently are important for supervisors to appreciate. And yet, no study has addressed what service users consider to be the outcomes that matter most from supervision.

\section{Implications for researchers}

In spite of the strong commitment in policy and practice to the use of supervision, the evidence base is surprisingly limited, almost all of it being correlational. The most obvious gap is in good evidence that the implementation of clearly defined models of supervision in an organisation leads to improved outcomes for workers and better services for users and carers. There is no shortage of models of supervision, and so their costs and effectiveness should be tested and compared. Research on the skills and effectiveness of training supervisors in integrated teams as well as social care agencies should also be developed. 


\section{Useful links}

\author{
British Association of Social Workers (BASW) \\ The College of Social Work (CoSW) \\ BASW/CoSW have collaborated on a report \\ looking at their members' experience of \\ supervision, with a view to developing a code of \\ good practice for supervision in social work, with \\ special reference to social workers working in \\ multi-disciplinary teams: http://cdn.basw.co.uk/ \\ upload/basw_13955-1.pdf
}

\section{Care Council Wales}

Supervision and appraising well: a guide to effective supervision and appraisal: www.ccwales.org.uk/ supervising-and-appraising-well/

\section{Care Quality Commission (CQC)}

The CQC's Essential Standards of quality and safety include Outcomes 12 and 14 that state staff should be properly trained and supervised, and have the chance to develop and improve their skills: www.cqc.org.uk/organisations-we-regulate/ registering-first-time/essential-standards

\section{Department for Education (DfE)}

The defunct Children's Workforce Development Council (CWDC) produced a guide to developing an integrated approach to supervision in children's trusts: www.education.gov.uk/publications/ eOrderingDownload/IW115-2010.pdf

\section{Department of Health, Social Services and} Public Safety (DHSSPS)

In Northern Ireland there is a mandatory regional policy and guidance which sets out the minimum standards for supervision of social workers employed by health and social care trusts. Whilst primarily focussed on childcare workers, it is broadly adopted for all social workers, It states that supervision must be delivered monthly with sessions of 1.5-2 hours in duration, with enhanced recommendations for newly qualified workers. www.dhsspsni.gov.uk/supervision_policy standards_and_criteria__regional_policy_for_ northern_ireland_health_and_social_care_ trusts.pdf
Local Government Association (LGA) In response to evidence submitted to the Social Work Task Force, the Standards for employers and supervision framework builds on existing guidelines for employers of social workers: www.local.gov.uk/c/document_library/get_file ?uuid=7e6d2140-fc0e-47cd-8b2f-2375812700 ad\&groupld=10171

\section{Skills for Care}

This workforce development tool has been produced by Skills for Care and the now defunct CWDC to promote the widespread provision of high-quality supervision across adult and children's social care:

www.skillsforcare.org.uk/home/home.aspx

\section{Related SCIE resources}

Guide 1: Managing practice www.scie.org.uk/publications/guides/guide01/

Care Skillsbase www.scie.org.uk/workforce/careskillsbase/

Guide 24: Learning together to safeguard children: developing a multi-agency systems approach for case reviews www.scie.org.uk/publications/guides/guide24/ index.asp

Guide 27: Leading practice: a development programme for first-line managers www.scie.org.uk/publications/guides/guide27/

Guide 38: Social care governance: a workbook based on practice in England www.scie.org.uk/publications/guides/guide38/

Knowledge review 16: Improving social and health care services www.scie.org.uk/publications/knowledge reviews/kr16.asph

Learning organisations pack www.scie.org.uk/publications/learningorgs/ index.asp 


\section{References}

1. Laming, H. (2009) The protection of children in England: a progress report, London: TSO.

2. Munro, E. (2011) The Munro review of child protection: final report - a child-centred system, London: DfE.

3. Noble, C. and Irwin, J. (2009) 'Social work supervision: an exploration of the current challenges in a rapidly changing social, economic and political environment', Journal of Social Work, vol 9, no 3, pp 345-358.

4. Department for Children School and Families (DCSF) (2009) The protection of children in England - action plan: the government's response to Lord Laming, London: TSO.

5. Davys, A. and Beddoe, L. (2010) Best practice in supervision: a guide for the helping professions, London: Jessica Kingsley.

6. Department of Health (DH) (2003) Care homes for older people, national minimum standards, London: $\mathrm{DH}$.

7. Care Quality Commission (CQC) (2010) Summary of regulations, outcomes and judgement framework, London: CQC.

8. Cameron, A. et al. (2012) Factors that promote and hinder joint working in health and social care, London: Social Care Institute for London.

9. Payne, M. (1996) What is professional social work? Birmingham: Venture Press.

10. Kadushin, A. (2002) Supervision in social work, New York: Columbia University Press.

11. Milne, D. (2010) 'The systematic review as an empirical approach to improving CBT supervision', International Journal of Cognitive Therapy, vol 3, no 3, pp 278-294.

12. Kavanagh, D.J. et al. (2003) 'Supervision practices in allied mental health: relationships of supervision characteristics to perceived impact and job satisfaction', Mental Health Services Research, vol 5, no 4, pp 187-195.
13. Renner, L.M., Porter, R.L. and Preister, S. (2009) 'Improving the retention of child welfare workers by strengthening skills and increasing support for supervisors', Child Welfare, vol 88, no 5, pp 109-127.

14. Bogo, M. et al. (2011) 'Supporting front-line practitioners' professional development and job satisfaction in mental health and addiction', Journal of Interprofessional Care, vol 25, no 3, pp 209-214.

15. Cole, D., Panchanadeswaran, S. and Daining, C. (2004) 'Predictors of job satisfaction of licensed social workers: perceived efficacy as a mediator of the relationship between workload and job satisfaction', Journal of Social Service Research, vol 31, no 1, pp 1-12.

16. Spence, S.H. et al. (2001) 'Clinical supervision in four mental health professions: a review of the evidence', Behaviour Change, vol 18, no 3, pp 135-155.

17. Bogo, M. and McKnight, K. (2006) 'Clinical supervision in social work: a review of the research literature', The Clinical Supervisor, vol 24, nos 1-2, pp 49-67.

18. Mor Barak, M.E. et al. (2009) 'The impact of supervision on worker outcomes: a metaanalysis', Social Service Review, vol 83, no 1, Pp 3-32.

19. Gibbs, J. (2001) 'Maintaining front-line workers in child protection: a case for refocusing supervision', Child Abuse Review, no 10, pp 323-335.

20. Allen, R.I. et al. (2004) 'The impact of job characteristics on social and human service workers', Social Work and Society, vol 2, no 2, PP 173-188.

21. Chenot, D., Benton, A.D. and Kim, H. (2009) 'The influence of supervisor support, peer support, and organizational culture among early career social workers in child welfare services', Child Welfare, vol 88, no 5, pP 129-147.

22. Lietz, C.A. (2008) 'Implementation of group supervision in child welfare: findings from 
Arizona's supervision circle project', Child Welfare, vol 87, no 6, pp 31-48.

23. Smith, C.H., Russell, R. and Giddings, M.M. (2007) 'Evaluating a social work supervision model in a real-world child welfare setting', Professional Development: The International Journal of Continuing Social Work Education, vol 10, no 1, pp 10-24.

24. Lee, J., Weaver, C. and Hrostowski, S. (2011) 'Psychological empowerment and child welfare worker outcomes: a path analysis', Child and Youth Care Forum, vol 40, no 6, pp 479-497.

25. Strand, V.C. and Dore, M.M. (2009) 'Job satisfaction in a stable state child welfare workforce: implications for staff retention', Children and Youth Services Review, vol 31, no 3, pp 391-397.

26. Barth, R.P. et al. (2008) 'Child welfare worker characteristics and job satisfaction: a national study', Social Work, vol 53, no 3, pp 199-209.

27. Ben-Porat, A. and Itzhaky, H. (2011) 'The contribution of training and supervision to perceived role competence, secondary traumatization, and burnout among domestic violence therapists', The Clinical Supervisor, vol 30, no 1, pp 95-108.

28. Bogo, M. et al. (2011) 'Interprofessional clinical supervision in mental health and addiction: toward identifying common elements', The Clinical Supervisor, vol 30, no 1, pp 124-140.

29. DeLoach, R. and Monroe, J. (2004) 'Job satisfaction among hospice workers: what managers need to know', Health Care Manager, vol 23, no 3, pp 209-219.

30. Egan, M. and Kadushin, G. (2004) 'Job satisfaction of home health social workers in the environment of cost containment', Health and Social Work, vol 29, no 4, pp 287-296.

31. Landsman, M.J. (2008) 'Pathways to organizational commitment', Administration in Social Work, vol 32, no 2, pp 105-132.
32. Kim, H. and Lee, S.Y. (2009) 'Supervisory communication, burnout, and turnover intention among social workers in health care settings', Social Work in Health Care, vol 48, no 4, pp 364-385.

33. Mena, K.C. and Bailey, J.D. (2007) 'The effects of the supervisory working alliance on worker outcomes', Journal of Social Service Research, vol 34, no 1, pp 55-65.

34. Chen, S.-Y. and Scannapieco, M. (2010) 'The influence of job satisfaction on child welfare worker's desire to stay: an examination of the interaction effect of self-efficacy and supportive supervision', Children and Youth Services Review, vol 32, no 4, pp 482-486.

35. Cearley, S. (2004) 'The power of supervision in child welfare services', Child and Youth Care Forum, vol 33, no 5, pp 313-327.

36. Allen, R.I. et al. (2004) 'The impact of job characteristics on social and human service workers', Social Work and Society, vol 2, no 2, PP 173-188.

37. Chenot, D., Benton, A.D. and Kim, H. (2009) 'The influence of supervisor support, peer support, and organizational culture among early career social workers in child welfare services', Child Welfare, vol 88, no 5, pp 129-147.

38. Smith, B.D. (2005) 'Job retention in child welfare: effects of perceived organizational support, supervisor support, and intrinsic job value', Children and Youth Services Review, vol 27, no 2, pp 153-169.

39. Nissly, J.A., Mor Barak, M.E. and Levin, A. (2005) 'Stress, social support, and workers' intentions to leave their jobs in public child welfare', Administration in Social Work, vol 29, no 1, pp 79-100.

40. Schudrich, W. et al. (2012) 'Factors impacting intention to leave in social workers and child care workers employed at voluntary agencies', Children and Youth Services Review, vol 34, no 1, pp 84-90. 
41. Simons, K.V. and Jankowski, T.B. (2007) 'Factors influencing nursing home social workers' intentions to quit employment', Administration in Social Work, vol 32, no 1, pp 5-21.

42. Abu-Bader, S.H. (2000) 'Work satisfaction, burnout, and turnover among social workers in Israel: a causal diagram', International Journal of Social Welfare, vol 9, no 3, pp 191-200.

43. Boyas, J. and Wind, L.H. (2010) 'Employment-based social capital, job stress, and employee burnout: a public child welfare employee structural model', Children and Youth Services Review, vol 32, no 3, pp 380-388.

44. Gibbs, J. (2001) 'Maintaining front-line workers in child protection: A case for refocusing supervision', Child Abuse Review, no 10, pp 323-335.

45. Strolin-Goltzman, J. et al. (2007) 'The relationship between organizational characteristics and workforce turnover among rural, urban, and suburban public child welfare systems', Administration in Social Work, vol 32, no 1, pp 77-91.

46. Strolin-Goltzman, J. (2008) 'Should I stay or should I go? A comparison study of intention to leave among public child welfare systems with high and low turnover rates', Child Welfare, vol 87, no 4, pp 125-143.

47. McGowan, B.G., Auerbach, C. and Strolin-Goltzman, J.S. (2009) 'Turnover in the child welfare workforce: a different perspective', Journal of Social Service Research, vol 35, no 3, pp 228-235.

48. Brannon, D. et al. (2007) 'Job perceptions and intent to leave among direct care workers: evidence from the Better Jobs Better Care demonstration', The Gerontologist, vol 47, no 6, pp 820-829.

49. Fakunmoju, S. et al. (2010) 'Intention to leave a job: the role of individual factors, job tension, and supervisory support', Administration in Social Work, vol 34, no 4, pp 313-328.

50. Jacquet, S.E. et al. (2008) 'The role of supervision in the retention of public child welfare workers', Journal of Public Child Welfare, vol 1, no 3, pp 27-54.

51. Fleming, G. and Taylor, B.J. (2007) 'Battle on the home care front: perceptions of home care workers of factors influencing staff retention in Northern Ireland', Health and Social Care in the Community, vol 15, no 1, pp 67-76.

52. Juby, C. and Scannapieco, M. (2007) 'Characteristics of workload management in public child welfare agencies', Administration in Social Work, vol 31, no 3, pp 95-109.

53. Collins-Camargo, C. and Royse, D. (2010) 'A study of the relationships among effective supervision, organizational culture promoting evidence-based practice, and worker self-efficacy in public child welfare', Journal of Public Child Welfare, vol 4, no 1, PP 1-24.

54. Collins-Camargo, C. and Millar, K. (2010) 'The potential for a more clinical approach to child welfare supervision to promote practice and case outcomes: a qualitative study in four states', The Clinical Supervisor, vol 29, no 2, pp 164-187.

55. Gonzalez, R.P. et al. (2009) 'Exit interviews with departed child welfare workers: preliminary findings', Journal of Public Child Welfare, vol 3, no 1, pp 40-63.

56. Dickinson, N.S. and Perry, R.E. (2002) 'Factors influencing the retention of specially educated public child welfare workers', Journal of Health and Social Policy, vol 15, nos 3/4, pp 89-103.

57. Maertz Jr, C.P. et al. (2007) 'The effects of perceived organizational support and perceived supervisor support on employee 
turnover', Journal of Organizational Behavior, vol 28, no 8, pp 1059-1075.

58. Yankeelov, P.A. et al. (2009) 'Individual and organizational factors in job retention in Kentucky's child welfare agency', Children and Youth Services Review, vol 31, no 5, pp 547-554.

59. Morazes, J.L. et al. (2010) 'Views of speciallytrained child welfare social workers: a qualitative study of their motivations, perceptions, and retention', Qualitative Social Work, vol 9, no 2, pp 227-247.

60. Faller, K.C., Grabarek, M. and Ortega, R.M. (2010) 'Commitment to child welfare work: what predicts leaving and staying?' Children and Youth Services Review, vol 32, no 6, pP 840-846.
61. Holmes, L., Munro, E.R. and Soper, J. (2010) Calculating the cost and capacity implications for local authorities implementing the Laming (2009) recommendations, Loughborough: Centre for Child and Family Research, University of Loughborough.

62. Bourn, D. and Hafford-Letchfield, T. (2011) 'The role of social work professional supervision in conditions of uncertainty', The International Journal of Knowledge, Culture and Change Management, vol 10, no 9, pp. 41-56.

63. Yoo, J. (2002) 'The relationship between organizational variables and client outcomes: a case study in child welfare', Administration in Social Work, vol 26, no 2, pp 39-61. 


\section{About the development of this product}

\section{Scoping and searching}

Focused searching was carried out between January and March 2012. The searches looked for empirical studies on the association between the process of supervision and outcomes for service users, workers and organisations. Searches addressed both children and adult's social work and social care, including joint and integrated settings.

This research briefing identifies empirical studies that report on the association between the process of supervision and outcomes for service users, workers and organisations. Intervention studies are included. The methods used to identify and organise material in this briefing were developed by the Social Care Institute for Excellence (SCIE). These involved undertaking systematic and reproducible searches of the research literature, identifying relevant studies and assessing their quality. Empirical data were extracted using a structured pro-forma which focused on various outcomes of supervision.

\section{Peer review and testing}

The authors have research and topic expertise. The briefing was peer reviewed internally for methodology. It was peer reviewed externally by Professor Marion Bogo, Faculty of Social Work, University of Toronto, Canada. We are grateful for her comments.

\section{About SCIE research briefings}

SCIE research briefings provide a concise summary of recent research into a particular topic and signpost routes to further information. They are designed to provide research evidence in an accessible format to a varied audience, including health and social care practitioners, students, managers and policy-makers. They have been undertaken using methodology developed by SCIE.

The information on which the briefings are based is drawn from relevant electronic databases, journals and texts, and where appropriate, from alternative sources, such as inspection reports and annual reviews as identified by the authors. The briefings do not provide a definitive statement of all evidence on a particular issue. SCIE research briefing methodology was followed throughout (inclusion criteria; material not comprehensively quality assured; evidence synthesised and key messages formulated by author): for full details, see www.scie.org.uk/publications/briefings/methodology.asp

SCIE research briefings are designed to be used online, with links to documents and other organisations' websites. To access this research briefing in full, and to find other publications, visit www.scie.org.uk/publications/briefings/ 


\section{Latest SCIE research briefings}

20 The implementation of individual budget schemes in adult social care

21 Identification of deafblind dual sensory impairment in older people

22 Obstacles to using and providing rural social care

23 Stress and resilience factors in parents with mental health problems and their children

24 Experiences of children and young people caring for a parent with a mental health problem

25 Children's and young people's experiences of domestic violence involving adults in a parenting role

26 Mental health and social work

27 Factors that assist early identification of children in need in integrated or inter-agency settings

28 Assistive technology and older people

29 Black and minority ethnic parents with mental health problems and their children

30 The relationship between dual diagnosis: substance misuse and dealing with mental health issues

31 Co-production: an emerging evidence base for adult social care transformation
32 Access to social care and support for adults with autistic spectrum conditions (ASC)

33 The contribution of social work and social care to the reduction of health inequalities: four case studies

34 Communication training for care home workers: outcomes for older people, staff, families and friends

35 Black and minority ethnic people with dementia and their access to support and services

36 Reablement: a cost-effective route to better outcomes

37 Mental health service transitions for young people

38 Mental health, employment and the social care workforce

39 Preventing loneliness and social isolation: interventions and outcomes

40 End of life care for people with dementia living in care homes

41 Factors that promote and hinder joint and integrated working between health and social care services

42 Returning children home from public care

43. Effective supervision in social work and social care

A full list of SCIE research briefings can be found at www.scie.org.uk/publications

\section{Social Care}

Institute for Excellence

Fifth floor

2-4 Cockspur Street

London

tel: 02070247650

SW1Y 5BH

fax: 02070247651

www.scie.org.uk 